\title{
CRITERIOS DEL SENTIDO DE MOVIMIENTO EN ESPEJOS DE FRICCION: CLASIFICACION Y APLICACION A LOS GRANITOS CIZALLADOS DE LA SIERRA DE SAN VICENTE (SIERRA DE GREDOS)
}

\author{
M. Doblas (*)
}

\begin{abstract}
RESUMEN
En este trabajo establecemos primeramente una clasificación de los diferentes criterios para la deducción del sentido de movimiento en espejos de fricción, obteniendo así una guía práctica de utilización en el campo o en el laboratorio, inexistente hasta ahora.

En segundo lugar, aplicamos algunos de estos criterios a los planos de cizallamiento $\mathrm{C}$ de los granitos deformados de la Sierra de San Vicente. Estas estructuras planares en granitos, que hasta ahora no se habían empleado en estudios de espejos de fricción, resultan muy útiles para comprobar y/o proponer criterios, ya que sus sentidos de movimiento se conocen en nuestra zona por investigaciones anteriores (Doblas et al., 1983, Doblas 1985). Una innovación que aportamos aquí, es la utilización del microscopio electrónico de barrido para el estudio de estas estructuras. Las estructuras observadas en los planos $C$, además de indicarnos el sentido del movimiento, revelan también interesantes características genéticas de los mismos.
\end{abstract}

Palabras clave: Espejos de fricción, sentido del movimiento, granitos deformados SC.

\begin{abstract}
In this paper, we first classify the different shearing sense criteria on slickensided surfaces, obtaining thus a practical guide both for the field, and the laboratory.

We then apply some of these criteria to the $C$ shearing planes present in the San Vicente deformed granites. These planar structures in granites, which have not been used yet for the study of slickensided surfaces, seem ideal for verifying and/or proposing criteria, as their shearing senses are already known from previous studies (Doblas et al., 1983, Doblas 1985). Some of these features are investigated here under the scanning electron microscope. Apart from indicative of the shearing senses, these structures also reveal interesting characteristics of formation of the $\mathrm{C}$ planes.
\end{abstract}

Key words: Slickensided surfaces, shearing senses, SC sheared granitoids.

\section{Introducción}

Las estructuras que permiten deducir el sentido del movimiento en espejos de fricción abundan en la bibliografía. Sin embargo, a la hora de utilizarlas prácticamente, resulta casi imposible hacerlo con eficacia ya que los criterios, además de estar dispersos, son confusos, e incluso a veces se contradicen unos a otros. Recientemente, Petit et al., (1983) proponen una sistemática para la utilización de estas estructuras, pero no abarcan más que unos pocos criterios, de los muchos existentes.

Por todo ello, nos pareció necesario establecer una clasificación completa y actualizada que incluyera todos los diferentes criterios del sentido de movimiento en espejos de fricción. Así, dividimos estas estructuras en dos grandes grupos, y estos a su vez en cinco subgrupos, obteniendo al final 34 criterios diferentes. Una de las aportaciones de este trabajo, es establecer cuales son los criterios más útiles. 
En trabajos anteriores (Doblas et al., 1983; Doblas, 1985), se estudiaron en la Sierra de San Vicente unos granitos deformados con planos sigmoidales S y planos de cizallamiento C, pudiéndose comprobar que estos últimos actúan bajo regímenes frágiles-dúctiles, produciendo espejos de fricción con abundantes estructuras. Ahora, investigamos los planos de cizallamiento $\mathrm{C}$ mediante la lupa binocular y el microscopio electrónico de barrido. Estos planos resultan ideales para el estudio y comprobación de criterios de movimiento en espejos de fricción, ya que en ellos el sentido del movimiento se conoce de antemano por otros criterios (Doblas et al., 1983; Doblas, 1985).

Además de ofrecernos la posibilidad de estudiar criterios del sentido de movimiento, las estructuras presentes en los planos $\mathrm{C}$ nos indican ciertas condiciones genéticas de formación de los mismos, como la existencia de un régimen frágildúctil con movimientos sísmicos ocasionales de tipo "stick-slip".

Este trabajo es una contribución en el estudio del sentido de movimiento en espejos de fricción, estableciendo una guía práctica de utilización de los diferentes criterios, y mostrando como se pueden aplicar estos, no solo a las fallas frágiles, sino también a los planos de cizallamiento $\mathrm{C}$ frágilesdúctiles de los granitos.

\section{Clasificación de los criterios del sentido de movimiento en espejos de fricción}

De las estructuras presentes en los espejos de fricción, se pueden deducir dos parámetros esenciales del movimiento: la dirección y el sentido.

Las estrías en el plano de fricción definen una lineación que indica la dirección del movimiento, y se deben en general a la acción abrasiva de un labio de la falla sobre el otro. Existen, sin embargo, algunas consideraciones a tener en cuentà dentro de este esquema general. Las lineaciones se producen a veces por recristalizaciones neoblásticas de minerales en el plano de falla (Durney \& Ramsay, 1973). Como señalan estos autores, cuando existen varios juegos de estas lineaciones, es a veces muy difícil deducir cual fue el último movimiento, ya que las recristalizaciones pueden ser sintaxiales o antitaxiales, y ambas indican órdenes diferentes. Según Petit et al., (1983), las estrías pueden no hallarse en el plano mismo del movimiento, sino en fracturas regularmente espaciadas, oblícuas al plano de la falla. Por último, los movimientos en los espejos de fricción resultan a veces mucho más complejos que las simples translaciones lineales que se describen normalmente, como lo demuestra la presencia ocasional de estrías "en guirnaldas" (Vialon et al., 1976).

Existen numerosos criterios que han sido utilizados para deducir el sentido del movimiento en espejos de fricción, y en la figura 1 proponemos una clasificación de los mismos. El reconocimiento del sentido de movimiento se basa en el carácter asimétrico que presentan estas estructuras, pudiéndose distinguir dos grandes grupos: i) estructuras asimétricas en el plano de fricción, y ii) estructuras asimétricas en secciones perperidiculares al plano de fricción, en dirección paralela a las estrías. Los diferentes criterios se pueden a su vez subdividir en cinco subgrupos, que se representan en la figura 1 ( $a, b, c, d$ y e), esquematizados en planta o en sección según cada caso.

El primer grupo (Fig. 1a), corresponde a estructuras en forma de $\mathrm{V}$ o de media luna en el plano de fricción, que nos indican el movimiento por la orientación del ángulo agudo que definen. En ciertos casos, este ángulo está orientado en sentido contrario al movimiento del labio opuesto (Fig. 1a, n." 1, 2 y 3): n." 1: colas ("trails"), que son zonas de material autóctono protegidas de la abrasión por detrás de protuberancias (Willis \& Willis, 1934; Tjia, 1967, Norris \& Barron, 1969; Gay, 1970); n." 2: escalones lunulares (Tjia, 1967); n." 3: microfracturas inversas (Dzulynski \& Kotlarczyk, 1965; Tjia, 1967). En otros casos, sin embargo, el ángulo agudo se orienta en el mismo sentido que el movimiento del labio opuesto de la falla (Fig. 1a, n. ${ }^{\circ} 4,5,6$ y 7): N. ${ }^{\circ} 4$ : surcos de rodamiento ("gouging-grain grooves"), producidas por el rodamiento de granos sobre la superficie de fricción (Willis \& Willis 1934; Tjia, 1967; Gay, 1970; Engelder, 1974 a, b; Jackson \& Dunn 1974); n. "5: espolones ("spurs"), que son zonas de acreción de material brechificado alóctono por delante de protuberancias (Willis \& Willis, 1934; Tjia, 1967); n." 6: lúnulas de rodamiento ("chatter-marks"), producidas por el rodamiento de granos sobre la superficie de fricción (Tjia, 1967; Wardlaw, et al., 1969); n. ${ }^{\circ}$ 7: canales tectónicos ("tectonic flutes"), debidos a indentaciones (Dzulynski \& Kotlarczyk, 1965). Finalmente, hay algunos casos en los cuales el ángulo agudo que definen las estructuras en $\mathrm{V}$ o en media luna puede tener cualquiera de las dos orientaciones anteriores (Fig. 1a, n. 8 y 9): n. ${ }^{\circ} 8$ : muescas ("scratches" y "bruises"), producidas por arañazos y abrasión (Willis \& Willis, 1934; Tjia, 1967; Jackson \& Dunn, 1974; Vialon et al., 1976); n. ${ }^{\circ} 9$ : microfracturas normales (Harris Jr., 1943; Dzulyns- 


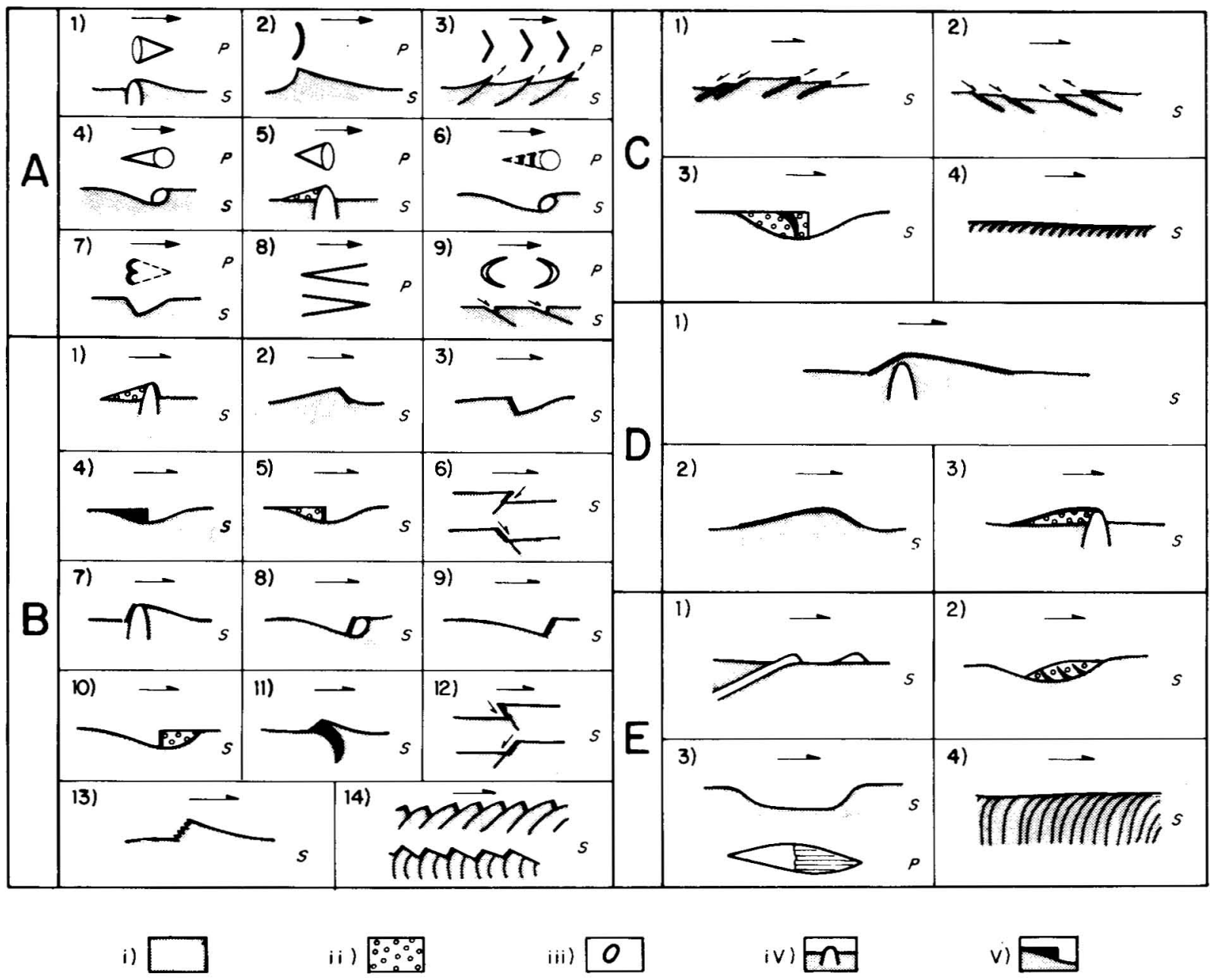

Fig. 1.-Clasificación de los diferentes criterios existentes para deducir el sentido del movimiento en espejos de fricción (ver explicaciones en el texto).

En cada apartado se resaltan en negro los criterios utilizados.

S: sección perpendicular al plano de fricción en dirección de las estrías. P: plano de fricción visto en planta. Las flechas indican el movimiento del labio opuesto de la falla, en planta (enteras), o en sección (partidas). Simbología utilizada en las secciones: i)-material autóctono; ii)-material alóctono; iii)-grano móvil rotando sobre la superficie de fricción; iv)-protuberancias en la superficie de fricción; v)-material recristalizado.

ki, 1953; Wegman \& schaer, 1957; Paterson, 1958).

El segundo grupo (Fig. 1b), corresponde a escalones en secciones perpendiculares al plano de fricción en dirección de las estrías, que indican el movimiento por la orientación de la cara de los mismos. Estos escalones sirvieron para definir el clásico "criterio de rugosidad", que fue aplicado durante mucho tiempo por los geólogos (el movimiento del bloque ausente de la falla se suponía era en sentido contrario a la rugosidad detectable con la palma de la mano). Aunque Tjia (1964) expuso claramente la falsedad de este criterio, antes que él, Harris Jr. (1943), Dzulynski (1953) y Paterson (1958), ya mostraron escalones con orientaciones que lo contradecían. En palabras de Paterson (1958), "el sentido del movimiento en una falla, no puede deducirse correctamente como lo hacen clásicamente los geólogos estructurales, es decir, observando la orientación de los escalones en los espejos de falla". Como se demuestra en la figura 1(b), la cuestión resulta muy complicada, ya que existen 14 tipos diferentes de escalones, 7 de los cuales, únicamente, obedecen el criterio de rugosidad. Los casos en los cuales los escalones obedecen el criterio de rugosidad, es decir que se orientan a favor del movimiento del labio opuesto de la falla, son los siguientes (Fig. 1b, n. ${ }^{\circ} 1,2,3,4,5$ y 6): n. ${ }^{\circ} 1$ : espolones (Tjia 1967); n. ${ }^{\circ}$ 2: montículos aborregados 
("knobby elevations"; Dzulynski \& Kotlarczyk, 1965); n." 3: escalones de arranque congruentes, es decir que miran a favor del movimiento, y que se producen por arranque brusco de material (Dzulynksi \& Kotlarczyk, 1965; Norris \& Barron, 1969, Vialon et al., 1976; Rod, 1966); n. ${ }^{\circ} 4$ : escalones de cristalización, debidos al crecimiento de minerales en zonas "de sombra" (Hobbs et al., 1976; Vialon et al., 1976; Petit et al., 1983); n. 5: escalones de acreción congruentes, producidos por apilamiento de material alóctono brechificado en zonas protegidas (Norris \& Barron, 1969); n. ${ }^{\circ} 6$ : microfracturas congruentes sintéticas, este último término indicando que estas microfracturas tienen el mismo movimiento que la falla, independientemente de su inclinación (Tjia, 1967; Norris \& Barron, 1969; Vialon et al., 1976). Los escalones se orientan en contra del movimiento del labio opuesto de la falla en otros casos (Fig. 1b, n." $7,8,9,10,11,12$ y 13): n. ${ }^{\circ} 7$ : colas (Willis \& Willis, 1934); n. ${ }^{\circ}$ 8: surcos de rodamiento (Petit et al., 1983); n. 9: escalones de arranque incongruentes, es decir orientados en contra del movimiento (Norris \& Barron, 1969; Riecker, 1965); n. ${ }^{\circ}$ 10: escalones de acreción incongruentes (Dzulynski \& Kotlarczyk, 1965; Tjia, 1967; Norris \& Barron, 1969); n. ${ }^{\circ} 11$ : grietas de tensión (Dzulynski \& Kotlarczyk, 1965); n. ${ }^{\circ} 12$ : microfracturas incongruentes antitéticas, es decir con escalones orientados en contra del movimiento, y sentido contrario a la falla (Harris Jr, 1943; Currie, 1969); n. ${ }^{\circ}$ 13: escalones estilolitizados (Arthaud \& Mattauer, 1969). Ciertos escalones producidos por la transposición de foliaciones previas en el plano de fricción, pueden orientarse en cualquiera de las dos direcciones ( . $^{\circ} 14$; Vialon et al., 1976).

El tercer grupo (Fig. 1c), corresponde a microfracturas en secciones perpendiculares al plano de fricción en dirección de las estrías, que indican el movimiento por su inclinación en estas secciones. Se citan en este sentido microfracturas normales $o$ inversas buzando en contra del movimiento ( $\mathrm{n}$. 1; Tjia, 1967; Norris \& Barron, 1969), o a favor del mismo (n. ${ }^{\circ}$; Tjia, 1967; Norris \& Barron, 1969; Friedman \& Logan, 1970; Conrad \& Friedman, 1976), grietas de tensión con formas en uña buzando hacia el movimiento (n. ${ }^{\circ} 3$; Dzulynski \& Kotlarczyk, 1965), o esquistosidades producidas por la falla que buzan en contra del movimiento (n.' 4; Petit et al., 1983).

El cuarto grupo (Fig. 1d), corresponde a montículos asimétricos en secciones perpendiculares al plano de fricción, en dirección de las estrías, que indican el sentido del movimiento por las orientaciones respectivas de los flancos cortos y largos de dichos montículos. El flanco corto estará orientado en contra del movimiento del labio opuesto de la falla, en el caso de las colas (n." 1; Gay, 1970), mientras que ocurre lo contrario en los montículos aborregados ( $\mathrm{n} .^{\circ} 2$ : Tjia, 1967), y los espolones (n. ${ }^{\circ} 3$; Tjia, 1967).

En el último grupo (Fig. 1e), se incluyen varios criterios no clasificables entre los anteriores, y que pueden estar tanto en el plano de fricción (n. ${ }^{\circ}$ ), como en secciones perpendiculares al mismo en la dirección de las estrías (n. ${ }^{\circ} 1,2$ y 4): n. ${ }^{\circ}$ 1: minerales arrastrados y desgarrados en la dirección del movimiento (Norris \& Barron, 1969); n." 2: escamas de material vítreo en forma de uña, producidas por deformaciones bruscas, y que buzan hacia el movimiento (Norris \& Barron, 1969); n. ${ }^{\circ}$ 3: surcos con pulidos diferenciales, siendo estos más acentuados en la mitad del surco orientado hacia el movimiento (Willis \& Willis, 1934); n. ${ }^{\circ}$ 4: efecto de arrastre de la falla sobre estructuras planares previas a la misma.

De la clasificación anteriormente expuesta, se deduce que la eficaz utilización de estos criterios, necesita de un exacto reconocimiento, en cada caso, del tipo que tenemos, y no basta pues con decir que son formas en $\mathrm{V}$, escalones, fracturas o montículos.

Uno de los resultados más interesantes que hemos obtenido mediante esta clasificación, es establecer cuales son los criterios más útiles para la deducción del sentido de movimiento en espejos de fricción. Ciertos criterios que se contradicen no son aconsejables a pesar de haber sido utilizados en la bibliografía. En el primer grupo (Fig. 1a) existen dos casos: formas en $V$ en muescas (n. ${ }^{\circ} 8$ ), y lúnulas en microfracturas normales (n. ${ }^{\circ}$ 9). En el segundo grupo (Fig. 1b), hay siete casos: escalones de arranque, ya que pueden ser tanto congruentes $\left(n .^{\circ} 3\right)$, como incongruentes (n. ${ }^{\circ}$ 9); lo mismo ocurre con los escalones de acreción ( $\mathrm{n} .^{\circ} 5$ y n. $\left.{ }^{\circ} 10\right)$, y con las microfracturas sintéticas y antitéticas $\left(\mathrm{n} .{ }^{\circ} 6 \mathrm{y} \mathrm{n} .^{\circ} 12\right)$; y las foliaciones previas transpuestas (n. $\left.{ }^{\circ} 14\right)$. Finalmente, en el tercer grupo (Fig. 1c), no es aconsejable utilizar la inclinación de las microfracturas, ya que pueden presentar cualquier buzamiento ( $\mathrm{n}^{\circ}$ 1 y n. 2 ).

\section{Aplicación a los granitos cizallados de la Sierra de San Vicente}

En trabajos anteriores (Doblas et al., 1983, Doblas, 1985), se estudiaron en la Sierra de San Vicente (Fig. 2), una serie de granitos deformados que presentan dos juegos de estructuras pla- 


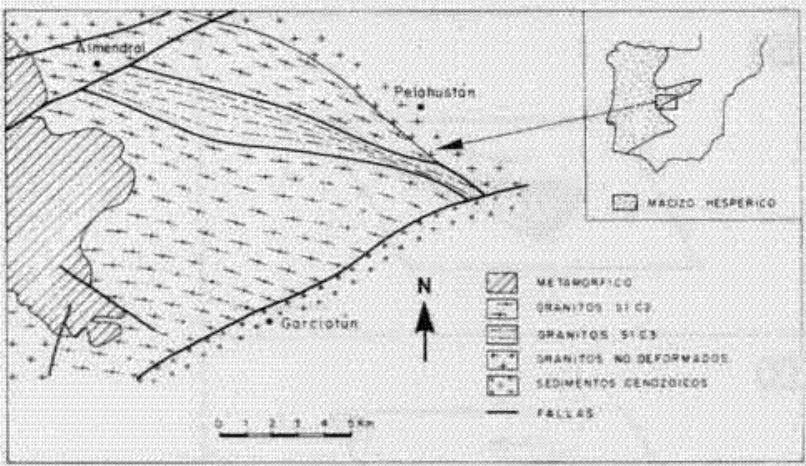

Fig. 2.-Esquema geológico de la Sierra de San Vicente,

nares, visibles en muestra de mano: planos $\mathrm{S}$ dúctiles y penetrativos, y planos de cizallamiento $\mathrm{C}$ frágiles-dúctiles, poco penetrativos, que deforman y cortan a los anteriores (Fig. 3a). Los planos $\mathrm{S}$ aparecen en todo el área, y representan el primer episodio deformativo de los granitos (S1). debido a los esfuerzos intrusivos del plutón sobre sus bordes, enlos contactos con las rocas de caja. Los planos S1 están cortados y deformados sigmoidalmente por los planos de cizallamiento C. más tardíos, los cuales son de dos tipos, y abarcan áreas diferentes: unos planos $\mathrm{C} 2$, que se encuentran en casi todo el área, con orientación WNW/ESE, buzamiento de $40^{\circ}$ al NNE, y estrías de fricción hacia el NNE, que indican un régimen tectónico distensivo hacia el NNE (inmediatamente posterior a $\mathrm{S} 1$ ); y unos planos $\mathrm{C} 3$, más tardíos que los anteriores, localizados en una banda WNW/ESE al N de la zona, verticales, con estrías de fricción horizontales, y que corresponden a un régimen tectónico transcurrente-sinestral. Según la presencia conjunta de los planos $\mathrm{S}$ y C, se distinguen así dos tipos de granitos deformados (Fig. 2): granitos $\mathrm{S} 1 \mathrm{C} 2$, y granitos $\mathrm{S} 1 \mathrm{C} 3$.

Los planos de cizallamiento tardíos $\mathrm{C}$ son muy vistosos en los afloramientos, llegando a producir resaltes escalonados (Fig. 3b), y desarrollando espejos de fricción (Fig. 3c). En este caso, la deformación, en vez de localizarse en un único plano de falla, se reparte en una serie regularmente espaciada de planos de cizallamiento $\mathrm{C}$, con desplazamiento pequeño en cada uno de ellos. La ventaja que presentan estos planos C para el estudio y/o comprobación de sentidos de movimiento en espejos de fricción, como los expuestos en el capítulo anterior, es que en ellos el sentido del movimiento se conoce ya previamente por otros criterios (Doblas et al., 1983; Doblas, 1985), como las relaciones $\mathrm{SC}$, las orientaciones oblicuas de los ejes C de los cuarzos, la deformación de ciertas venas leucocráticas (ver Fig. 3a), y la de-
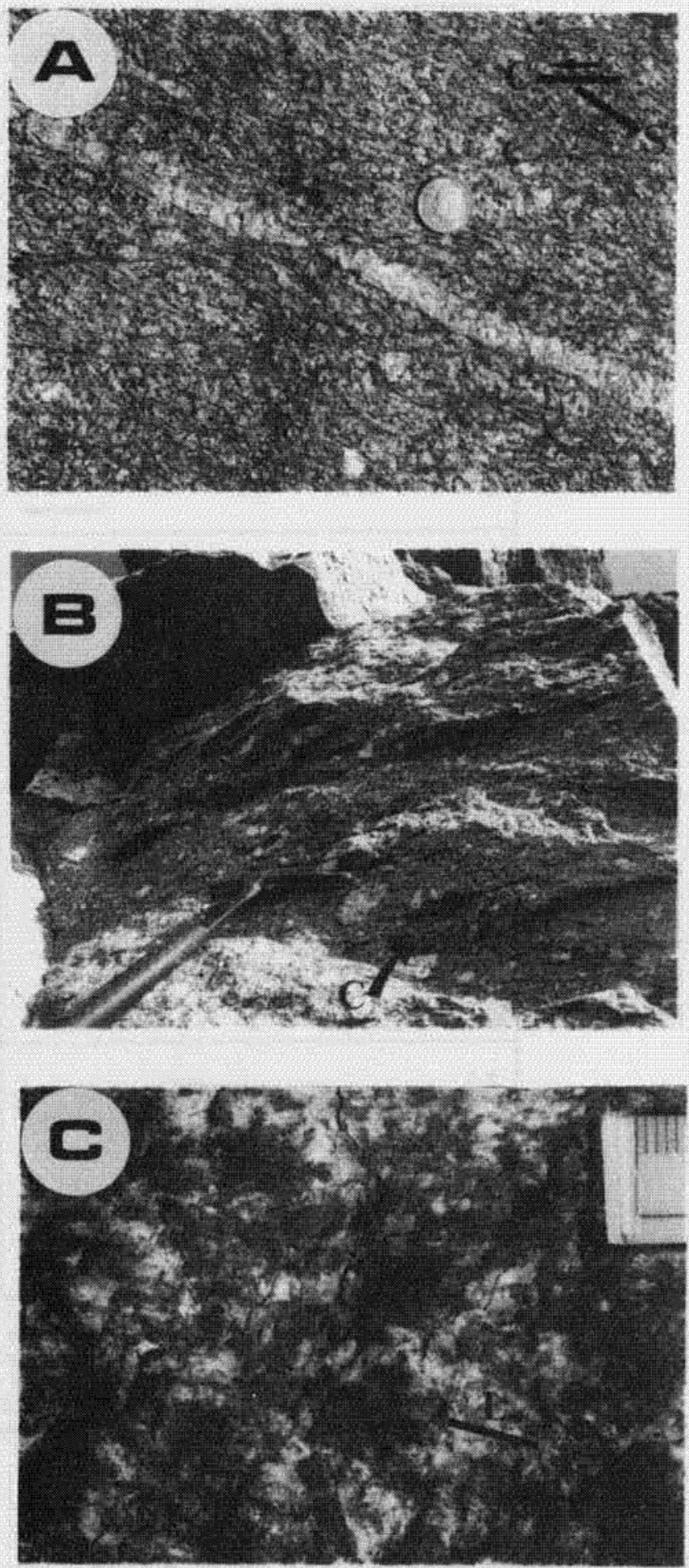

Fig. 3.-Granitos deformados de la Sierra de San Vicente: A)-Granito cizallado SC. B)-Plano $C$ con espejos de fricción en relieve, en un granito deformado SC. C)-Espejo de fricción en un plano $C$ (L:lineación). 


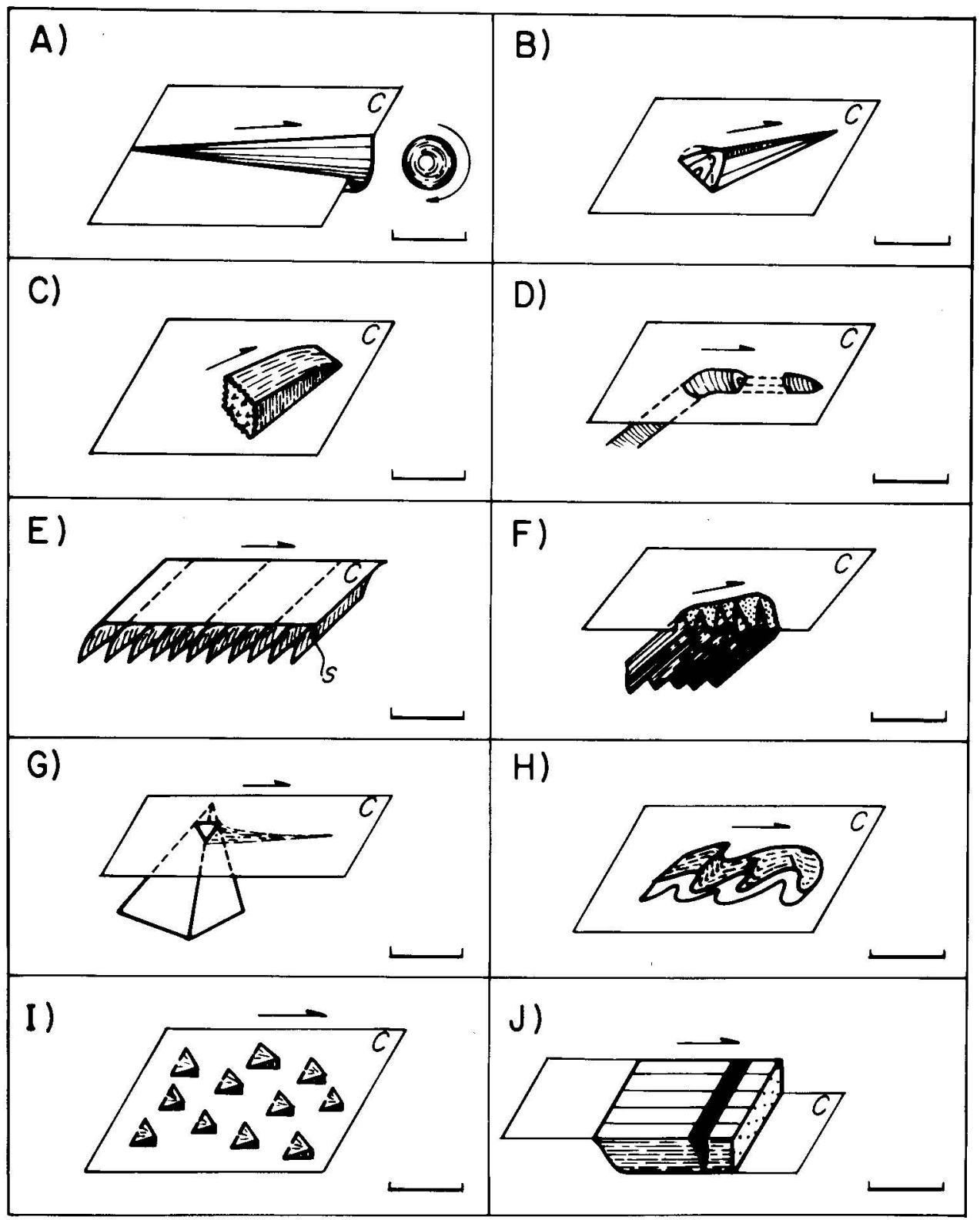

Fig. 4.-Representación esquemática de algunas de las estructuras observadas en los espejos de fricción de los planos C de los granitos deformados de la Sierra de San Vicente (ver explicaciones en el texto).

C: planos C; S: planos S; flechas: movimiento del bloque que falta; Escalas gráficas: $1 \mathrm{~mm}$. en $\mathrm{a}, \mathrm{b}, \mathrm{f}$, i y $1 \mathrm{~cm}$. en las demás.

formación de minerales a todas las escalas. En estos planos, las estrías se deben en general a recristalizaciones neoblásticas de micas, aunque existen también marcas de abrasión y de desgaste (Fig. 3c). En la figura 4 se representan esquemáticamente algunos de los ejemplos más relevantes, estudiados, o bien con el microscopio electrónico de barrido, o bien con la lupa binocular. En la figura 5 se muestran los cuatro casos estudiados con el microscopio electrónico de barrido (que aparecen esquematizados en las figuras 4 , a, $\mathrm{b}, \mathrm{f}, \mathrm{i})$. Este estudio fue realizado con el microscopio electrónico de barrido "Cameca MBX" de la Universidad de Harvard (EEUU), asistido por un ordenador "Tracor Northern", mediante el sistema de automatización "Sandia Task-83" (Chambers, 1983): este microscopio se utilizó en versión TEM ("Transmitted electron microscope"; Goldstein et al. 1981), obteniéndose las imágenes BSE ("Back-scattered electron") de la figu- 

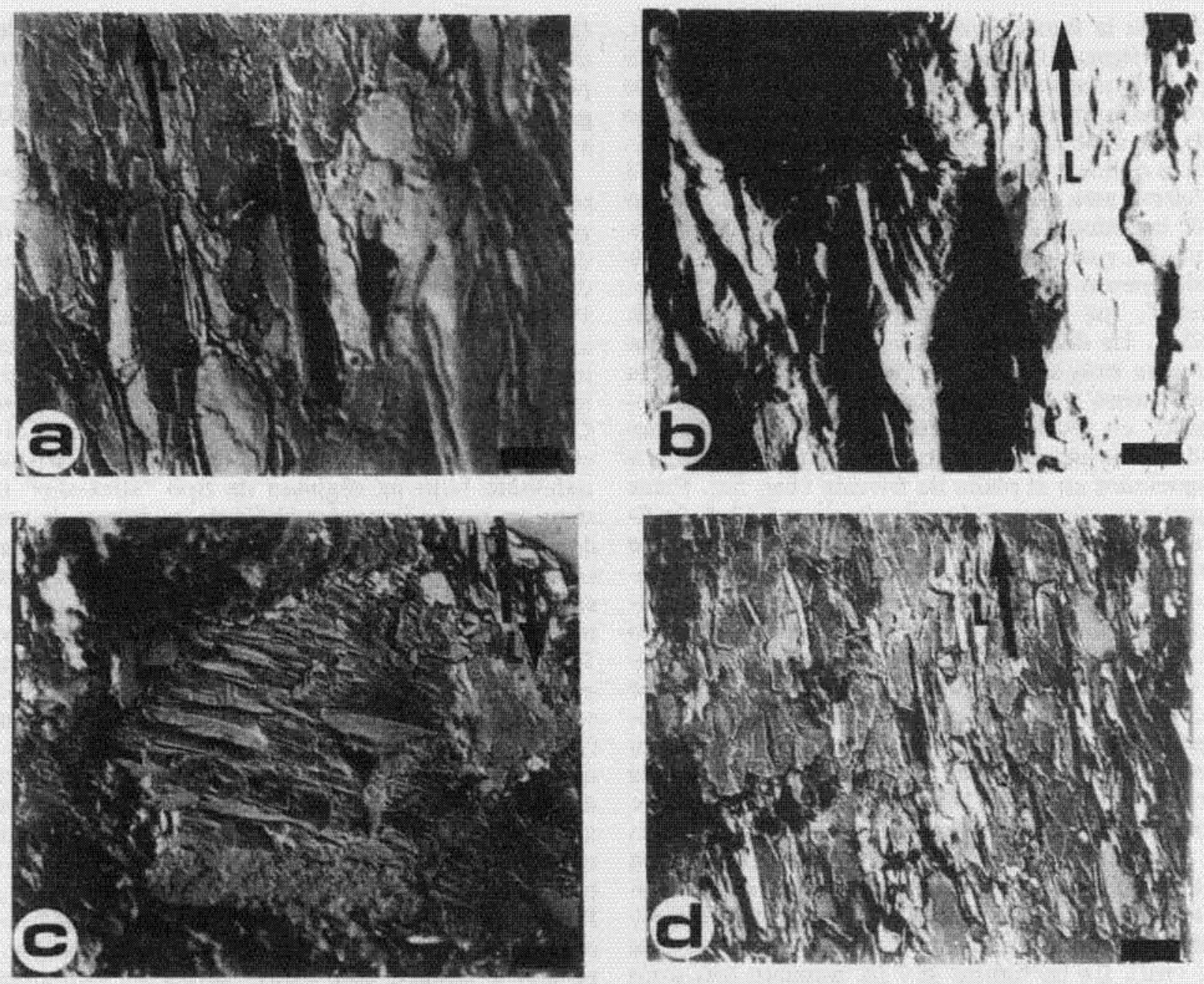

Fig. 5.-Imágenes "Back-scattered clectron" obtenidas con el microscopio electrónico de barrido (en tersion " lransmitted electron microscope"), de algunos de los criterios del sentido de movimiento observados en los planos C (ver texto para las explicaciones).

Flechas: movimiento del bloque visible. L: lineación en los planos C. Escalas: 38 um en a, 60 um en b, y 114 um en c. y en d. Todas las fotos están tomadas sobre los planos $\mathrm{C}$ vistos en planta.

ra 5. Esta técnica había sido empleada ya para el estudio de las deformaciones de ciertos minerales (Schedl et al, 1986), pero nunca para el estudio de estructuras de espejos de fricción, que nos permitan deducir el sentido del cizallamiento.

En la figura $4 a$, se representa un surco de rodamiento producido por un grano rotando en un plano $\mathrm{C}$, y corresponde a la interpretación de la imagen de la figura 5a. El criterio del sentido del movimiento utilizado aquí es el de una estructura en $\mathrm{V}$ en el plano de fricción, cuyo ángulo agudo está orientado en la dirección del movimiento del bloque que falta (Fig. 1a, n, ${ }^{\circ} 4$ ). Los casos del esquema de la figura $4 \mathrm{~b}$ y de la imagen de la figura $5 \mathrm{~b}$, corresponden a un escalón de arranque incongruente, y el criterio utilizado aquí es el de un escalón en sección perpendicular al plano de fricción en la dirección de las estrías, y que está orientado en contra del movimiento del bloque opuesto (Fig. 1b, n. ${ }^{\circ} 9$ ). En la figura $4 \mathrm{c}$, se representa un escalón estilolitizado producido en un mineral protuberante, que se orienta en contra del movimiento del bloque opuesto (Fig. 1b, ${ }^{\circ}{ }^{\circ}$ 13). El caso esquematizado en la figura $4 d$, corresponde a una lámina de mica arrastrada y desgarrada en la dirección del movimiento (Fig. 1e, n. ${ }^{\circ}$ 1). En la figura 4 e, se muestra el efecto de arrastre de los planos $\mathrm{S}$ a lo largo de los planos $C$, que se observa en sección perpendicular al plano de fricción (Fig. 1e, n. ${ }^{\circ} 4$ ). Los casos siguientes corresponden, o bien a una combinación de varios de los criterios expuestos en la clasifica- 
ción de la figura 1 , o bien a criterios diferentes. En la figura $4 \mathrm{f}$, tenemos un grano que ha rotado en la dirección del cizallamiento, a la vez que se rompía a modo de fichas de dominó, a lo largo de una serie espaciada y escalonada de microfracturas oblicuas a $\mathrm{C}$ (en la imagen de la figura $5 \mathrm{c}$ se observa este escalonamiento en planta). Como se vé, las microfracturas buzan en contra del movimiento, y su sentido es opuesto al del plano de cizallamiento (este criterio denominado de "shearsteps", fue propuesto por Simpson \& Schmid, 1983). La deducción del sentido del movimiento en este caso se basa en dos criterios (Fig. 4f): la inclinación y el movimiento de las "fichas de dominó" en sección perpendicular al plano de fricción, y la presencia de escalones incongruentes y espaciados en el plano de fricción (Fig. 5c). Estos escalones son parecidos al criterio n. ${ }^{\circ} 12$ de la figura 1(b), pero se diferencian en que aquí existe una rotación-fracturación del grano en su conjunto. El caso representado en la figura $4 \mathrm{~g}$ corresponde a un mineral cuya protuberancia ha sido machacada y arrastrada en la dirección del movimiento. Dos criterios nos indican aquí el sentido del movimiento: la dirección de arrastre del mineral (Fig. 1e, n. ${ }^{\circ}$ 1), y la forma en $\mathrm{V}$ en planta debida a este arrastre, y cuyo ángulo agudo está orientado en contra del movimiento del bloque opuesto. El caso representado en la figura $4 \mathrm{~h}$ es el de una lámina de mica que ha sido deformada en micropliegues asimétricos, que indican el movimiento por su forma en $\mathrm{Z}$ (este es un criterio clásicamente utilizado por los geólogos a la escala de visu). En las figuras $4 \mathrm{i}$ y $5 \mathrm{~d}$, tenemos una serie de microescalones de láminas de mica recristalizadas, en la dirección del movimiento del bloque que falta (Fig. 1b, n." 4). Existe además un criterio adicional, y es la presencia en planta de formas en $\mathrm{V}$ con el ángulo agudo orientado en contra del movimiento, debidas a "micro-fallas inversas-recristalizaciones" de las laminas de mica (Fig. 1a, n. ${ }^{\circ} 3$ ). Por último, en la figura $4 \mathrm{j}$ se pueden observar también dos criterios del sentido de movimiento diferentes: un escalón de cristalización mirando a favor del movimiento (Fig., 1b, n. $\left.{ }^{\circ} 4\right)$, asociado con una grieta de tensión en su borde, buzando hacia el movimiento (Fig. 1c, n. ${ }^{\circ}$ $3)$.

Como hemos visto, se pueden aplicar los criterios expuestos en nuestra clasificación a planos de cizallamiento $\mathrm{C}$ en granitos. Hasta ahora no se habían utilizado criterios del sentido de movimiento en espejos de fricción, aplicados a granitos SC: en nuestra zona se conocía ya de antemano el sentido del cizallamiento, pero en muchos otros granitos donde solo existan los planos C
(Doblas, 1985), el único criterio que se puede usar es este de los espejos de fricción. Esto abre por lo tanto, un campo de estudio interesante en granitos deformados SC, hasta ahora restringido a fallas frágiles.

Además de los sentidos del movimiento, podemos deducir también una serie de características genéticas de los granitos de nuestra zona, a partir de las estructuras en espejos de fricción observadas. Según varios autores (Norris \& Barron, 1969; Currie, 1969), la presencia de escalones de arranque, sucede bajo condiciones de presiones intermedias, correspondientes a regímenes frágiles-dúctiles, y esto es lo que ocurre en los planos $\mathrm{C}$ de la Sierra de San Vicente. Varios datos indican además que los planos $\mathrm{C}$ funcionaron ocasionalmente bajo un régimen de tipo "stick-slip" o sísmico: según Engelder (1974a), los surcos de rodamiento microscópicos como el de la figura 5a son típicos de este tipo de regímenes, a presiones superiores a 300 bars; lo mismo afirman en cuanto a las condiciones genéticas Riecker (1965) y Tjia (1967), para el caso de los escalones de arranque incongruentes como el de la figura 5b; según Engelder (1974a) y Jackson \& Dunn (1974), un alto contenido en cuarzo (como sucede en nuestras rocas) favorece el desplazamiento de tipo "stick-slip"; por último, en nuestra zona abundan las pseudotaquilitas de probable origen sísmico, tanto paralelas, como oblicuas a C (Doblas et al., 1983; Doblas, 1985; Doblas, 1987). Por lo tanto, de las estructuras en espejos de fricción en los planos C de la Sierra de San Vicente, podemos deducir que estos planos se formaron bajo condiciones frágiles-dúctiles, y con movimientos ocasionales de tipo "stick-slip" o sísmi$\cos$.

\section{Conclusiones}

En este trabajo hemos establecido una clasificación de los diferentes criterios existentes para la deducción del sentido de movimiento en espejos de fricción. Esta clasificación, que nos indica cuales son los criterios más útiles, es de fácil aplicación práctica para la geología de campo y de laboratorio.

Hemos utilizado algunos de estos criterios en los planos de cizalla $C$ de los granitos deformados de la Sierra de San Vicente. De este modo, se abre un nuevo campo de estudio de los espejos de fricción, hasta ahora no considerado: estas estructuras no se limitan a las clásicas fallas frágiles, sino que se dan también en condiciones de mayor ductilidad, y en particular tienen un gran 
interés y aplicabilidad a las vastas regiones graníticas donde existen a menudo granitos SC, granitos-gneisses, y ultramilonitas graníticas.

Las estructuras observadas en los planos $\mathrm{C}$ de los granitos deformados, nos indican además, que estos se formaron bajo condiciones frágiles-dúctiles, y con desplazamientos ocasionales de tipo "stick-slip" o sísmicos.

\section{AGRADECIMIENTOS}

Agradezco especialmente la colaboración de los Profesores R. Capote y A. Rivas (Universidad Complutense), así como los comentarios del Profesor B.P. Wernicke (Universidad de Harvard, EEUU), la ayuda con el microscopio electrónico de barrido del Dr. D. Lange de la Universidad de Harvard, el trabajo fotográfico de D.J.S. Corral, y la utilización de un ordenador para el tratamiento de textos de la empresa Honeywell S.A.

\section{Referencias}

Arthaud, F. y Mattauer, M.G. (1969). Exemples de stylolites d'origine téctonique dans le Languedoc, leurs relations avec la téctonique cassante. Bull. Soc. Geol. France, 7 (11), 738-744.

Chambers, W.F. (1983). Sandia Task. 83. An electron microprobe automation system. Sandia National Laboratories Studies, Albuquerque, New Mexico, 125 pág.

Conrad II, R.E. y Friedman, M. (1976). Microscopic feather fractures in the faulting process. Tectonophysics, 3, 187-198.

Currie, J.B. (1969). Comments on the paper "Structural analysis of features on natural and artificial faults" by Norris \& Barron. In: Research in tectonics, (Baer \& Norris ed.), Geol. Sur. Can. Pap., n. ${ }^{\circ}$ 68-52, 168-172.

Doblas, M., Capote, R. y Casquet, C. (1983). Fenómenos de cizalla dúctil en los granitoides de la Sierra de San Vicente (Sierra de Gredos). Stud. Geol. Salm., 18, 27-38.

Doblas, M. (1985). SC deformed rocks: the example of the Sierra de San Vicente sheared granitoids (Sierra de Gredos, Toledo, Spain). Tesis de Master de la Universidad de Harvard, Cambridge, EEUU, 145 pág.

Doblas, M. (1987). Paleoseismicity in the Spanish Central Range: Evidence from the pseudotachylites. Geogaceta, 2.

Durney, D.W. y Ramsay, J.G. (1973). Incremental strains measured by syntectonic crystal growths. In: Gravity \& Tectonics, (K.A. De Jong y R. Scholten ed.), Willey, 67-96.

Dzulynski, S. (1953). Tektonika pd. czesci wyzyny Krakowskiej. Act. Geol. Pol., 3, 325-440.

Dzulynski, S. y Kotlarczyk, J. (1965). Tectogliphs on slickensided surfaces. Bull. Ac. Pol. Sci., 13, 149-154.
Engelder, J.T. (1974a). Microscopic wear grooves on slickensides: indicators of paloseismicity. Jour. Geoph. Res., 79, 4.387-4.392.

Engelder, J.T. (1974b). Cataclasis and the generation of fault gouge. Geol. Soc. Am. Bull., 85, 1.515-1.522.

Friedman, M. y Logan, J.M. (1970). Microscopic feather fractures. Geol. Soc. Am. Bull., 81, 3.417-3.420.

Gay, N.C. (1970). The formation of step structures on slickensided shear surfaces. Jour. Geol., 78, 523-532.

Goldstein, J.I., Newbury, D.E., Echlin, P., Joy, D.C. Fiori, C. y Lifshin, E. (1981).Scanning electron microscopy and $x$-ray microanalysis. Plenum Press, 638 pág.

Harris Jr., S.E. (1943). Friction cracks and the direction of glacial movement. Jour. Geol., 51, 244-258.

Hobbs, B.E., Means, W.D. y Williams, P.F. (1976). An outline of structural geology. Willey, 571 pág.

Jackson, R.E. y Dunn, D.E. (1974). Experimental sliding friction and cataclasis of foliated rocks. Int. Jour. Rock Mech. Min. Sci. Geoq. Abs., 11, 235-249.

Norris, D.K. y Barron, K. (1969). Structural analysis of features on natural and artificial faults. In: Research in tectonics, (Baer \& Norris ed.), Geol. Sur. Can. Pap., n. ${ }^{\circ}$ 68-52, 136-167.

Paterson, M.S. (1958). Experimental deformation and faulting in wombeyan marble. Bull. Geol. Soc. Am., 69, 465-476.

Petit, J.P., Proust, F. y Tapponnier, P. (1983). Critères de sens de mouvement sur les miroirs de failles en roches non calcaires. Bull. Soc. Geol. Fra., 7, n. ${ }^{\circ} 4,589-608$.

Riecker, R.E. (1965). Fault-plane features: an alternative explanation. Jour. Sed. Petr., 35, 746-748.

Rod, E. (1966). A discussion of the paper "Fault-plane features: an alternative explanation" by R.E. Riecker. Jour. Sed. Petr., $36,1.163-1.165$.

Schedl, A., Kronemberg, A.K. y Tullis, J. (1986). Deformation microstructures of Barre granite: an optical SEM and TEM study. Tectonophysics, 122, 149-164.

Simpson, C. y Schmid, S.M. (1983). An evaluation of criteria to deduce the sense of movement in sheared rocks. Geol. Soc Am. Bull., 94, 1.281-1.288.

Tjia, H.D. (1964). Slickensides and fault movements. Geol. Soc. Am. Bull., 75, 683-686.

Tjia, H.D. (1967). Sense of fault displacemenst. Geol. Mijn., 46, 392-396.

Vialon, P., Ruhland, M. y Grolier, J. (1976). Eléments de téctonique analytique. Masson ed., 118 pág.

Wardlaw, N.C., Stauffer, M.R. y Hoque, M. (1969). Striations, giant grooves, and superposed drag folds, Interlake area, $\mathrm{Ma}$ nitoba. Can. Jour. Eart. Sc., 6, 577-593.

Wegman, E. y Schaer, J.P. (1957). Lunules téctoniques et traces de mouvements dans les plis du Jura. Eclog. Geol. Helv., $50(2), 491-496$

Willis, B. y Willis, R. (1934). Geologic structures. Mc. GrawHill, 420 pág.

Recibido el 12 de Febrero de 1987 Aceptado el 22 de Marzo de 1987 